\title{
Recuperación de Au a partir de PCB's en desuso por vía alternativa al proceso de cianuración.
}

\section{Gold recovery from wasted PCB's by a non-cyanide process.}

Presentación: 00/00/0000

\section{Doctorando:}

\section{Sebastián Camelino}

Universidad Tecnológica Nacional - Facultad Regional Córdoba

sebascamelino@gmail.com

\section{Director/es: \\ Mariana Minchiotti \\ Roberto Lucci}

\section{Resumen}

El presente trabajo resume las investigaciones experimentales concernientes a la puesta a punto de un proceso de extracción hidrometalúrgico que cumpla con la condición de no emplear ningún tipo de cianuro, y a su vez aplicable a placas de circuitos impresos. Es por ello que se ha trabajado con soluciones de agua regia, tiosulfato de amonio y de sodio y tiourea. Mediante la espectrofotometría UV/Vis, pudo ponerse a punto una técnica de análisis cuantitativo de $\mathrm{Au}$. También se exponen los resultados obtenidos en la adsorción/desorción con carbón activado en pruebas CIP (carbón en pulpa).

Palabras claves: Hidrometalurgia, Espectrofotometría UV/Vis, Lixiviación, Carbón activado, adsorción/desorción.

\begin{abstract}
The present work summarizes the experiences oriented to develop a hydrometallurgical process that will not need any kind of cyanide. It also has to be applicable to metals recovery from printed circuit boards. Therefore, solutions of aqua regia, ammonium thiosulphate, sodium thiosulphate and thiourea, were employed. By UV/Vis spectrophotometry it was able setting up an analisys cuantitative technique for gold detection. Besides it is exposed the results gathered after the CIP (carbon-in-pulp) adsortion/desortion tests.
\end{abstract}

Keywords: Hidrometallurgy, UV/Vis Spectrophotometry, Leaching, Activated coal, adsorption/desortion.

\section{Introducción}

El creciente desarrollo y la innovación tecnológica trae aparejadas consecuencias no solo en los niveles de confort, sino que también representa un importante pasivo ambiental debido a los recursos que son necesarios para la producción de artículos. Por otro lado, el material que entra en desuso, de alguna manera debe retornar al ciclo productivo para poder intentar satisfacer la tendencia global en cambiar los sistemas de producción lineal a sistemas de producción circular. En ese marco, resulta atractiva la búsqueda de procesos alternativos a los convencionales para el reaprovechamiento de tales fuentes secundarias de recursos.

\section{Objetivo general:}

Proponer un proceso hidrometalúrgico sustentable con el medio ambiente, adecuado para la recuperación del oro contenido de placas de circuitos impresos (PCB's) provenientes de chatarra electrónica.

\section{Objetivos específicos:}

I) Diseñar una metodología adecuada para la preparación y caracterización del material de partida; 
II) Plantear alguna técnica de análisis adecuada, confiable y económica para las distintas soluciones obtenidas a partir de la chatarra electrónica;

III) evaluar la influencia de distintos parámetros y condiciones para la disolución del oro en soluciones de tiosulfato de amonio;

III) establecer un procedimiento adecuado y conveniente para la extracción del oro desde las soluciones de tiosulfato de amonio.

\section{Materiales y métodos.}

\section{Esquema general}

Las placas de circuitos impresos (PCB's) por sus siglas en inglés constituyen una importante fuente secundaria de metales (tabla 1), de los cuales algunos pueden ser recuperados directamente por operaciones unitarias mientras que, para otros, resulta necesario el ulterior tratamiento con procesos unitario.

\begin{tabular}{|l|c|c|c|c|c|c|c|}
\hline \multirow{2}{*}{\multicolumn{1}{|c|}{ RAEE }} & \multicolumn{6}{c|}{ \% en peso } & \multicolumn{2}{c|}{ ppm } \\
\cline { 2 - 8 } & $\mathrm{Fe}$ & $\mathrm{Cu}$ & $\mathrm{Al}$ & $\mathrm{Sn}$ & $\mathrm{Pb}$ & $\mathrm{Ag}$ & $\mathrm{Au}$ \\
\hline PCB TV & 28 & 10 & 10 & 5 & 1 & 280 & 20 \\
\hline PCB PC & 7 & 20 & 5 & 8 & 1,5 & 1000 & 250 \\
\hline PCB Celulares & 5 & 13 & 1 & 0,4 & 0,3 & 1380 & 350 \\
\hline PCB (otras) & 12 & 10 & 7 & 2,5 & 1,2 & 280 & 110 \\
\hline Desechos TV & - & 3,4 & 1,2 & - & 0,2 & 20 & $<10$ \\
\hline Desechos DVD & 62 & 5 & 2 & - & 0,3 & 115 & 15 \\
\hline Desechos PC & 20 & 7 & 14 & 1,8 & 6 & 189 & 16 \\
\hline
\end{tabular}

Tabla 1: Contenidos metálicos recuperables según el origen de las PCB's.

En el esquema de la figura 1 se presenta de manera general el procedimiento llevado a cabo para la extracción de oro desde chatarras electrónicas.

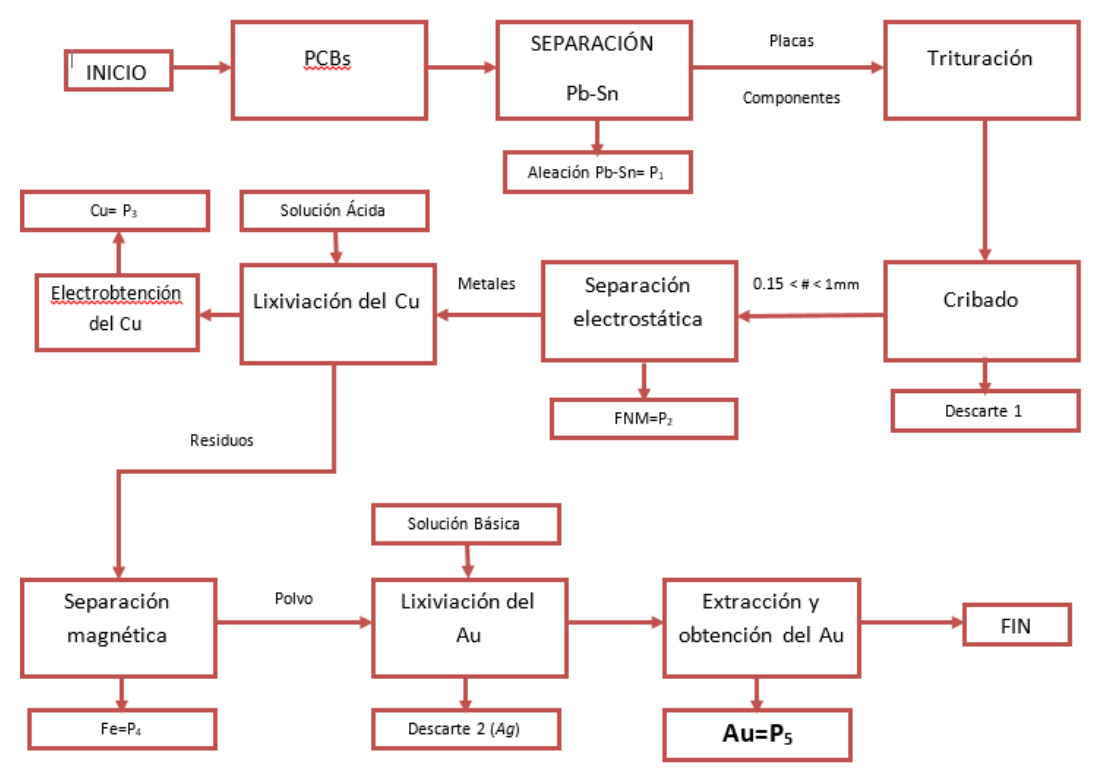

Figura 1: Esquema del proceso general de recuperación de metales a partir de placas de circuitos impresos provenientes de RAEE's,

Todos los reactivos empleados en las etapas hidrometalúrgicas son de grado analítico. Se utilizaron soluciones de tiosulfato de amonio y de sodio, hidróxido de amonio y de sodio, tiourea, ácidos sulfúrico, clorhídrico y nítrico (agua regia) y solventes de extracción orgánica. Para la caracterización se han empleado las técnicas de ICP-MS, ensayos de copelación, difracción de rayos X y espectrofotometría UV/Vis. 

auríferos.

La figura 2 esquematiza el procedimiento llevado a cabo para obtener una curva de calibración para la cuantificación de Au por espectrofotometría UV/Vis. Consistió fundamentalmente en la elaboración de diversas soluciones patrón a partir de una muestra de Au 99,6 \% y la evaluación de las curvas espectrofotométricas obtenidas.

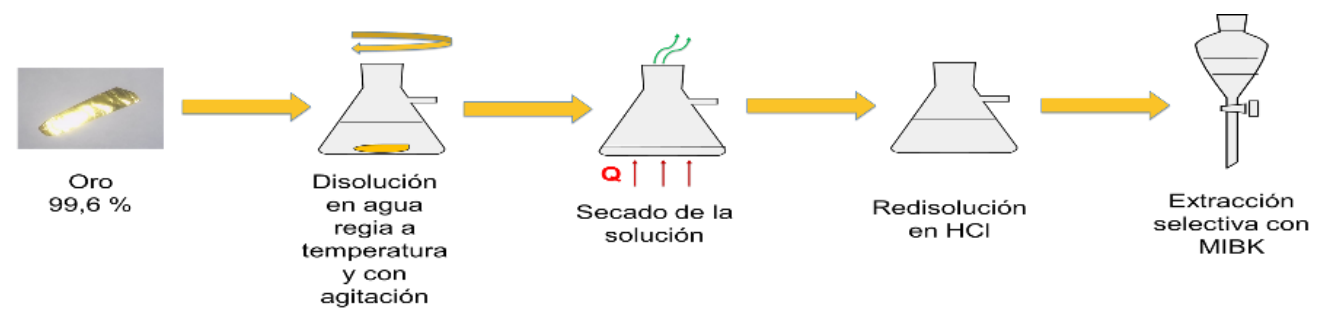

Figura 2 Procedimiento llevado a cabo para la disolución de cantidades controladas de Au a distintas concentraciones.

\section{Experiencias de carga y descarga con carbón activado.}

El polvo metálico de las placas, luego de haber pasado por todas las operaciones físicas e inclusive después de la lixiviación con $\mathrm{H}_{2} \mathrm{SO}_{4}$ (puesta en solución del $\mathrm{Cu}$, figura 1), es nuevamente lixiviado con soluciones de tiosulfato de amonio a concentraciones variables entre 0,01 a $0,2 \mathrm{M}$. A partir de estas últimas soluciones se realizan las pruebas CIP (carbón en pulpa) de adsorción/desorción en carbón activado (esquema en la figura 3).

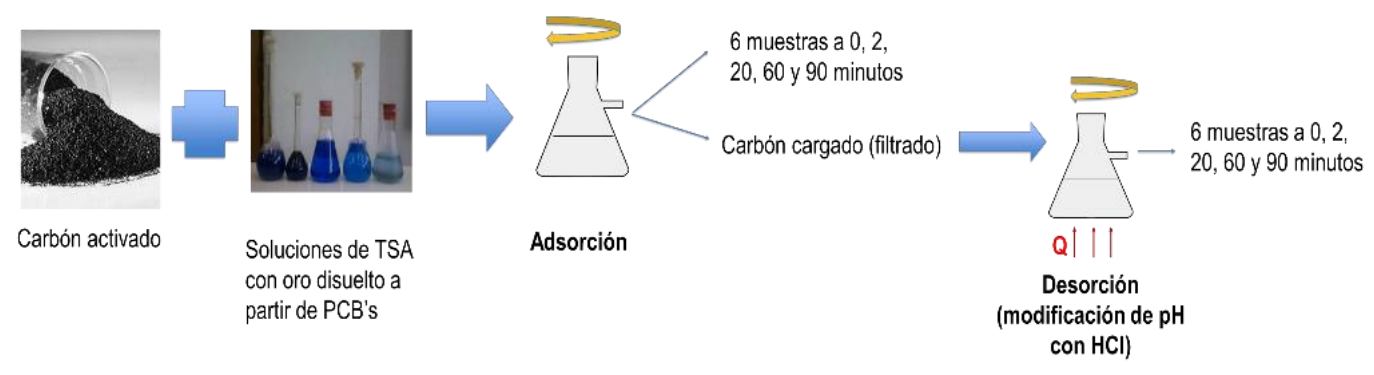

Figura 3 Procedimiento llevado a cabo en las pruebas de adsorción/desorción con carbón activado.

\section{Resultados}

\section{Curvas de calibración.}

En la Tabla 2 se pueden ver las mediciones obtenidas a $332 \mathrm{~nm}$ tanto para espectros de absorción medidos en absorbancia directa como derivativa de segundo orden.

\begin{tabular}{|c|c|c|}
\hline CONCENTRACIÓN [mM] & ABSORBANCIA A 332 nM & SEGUNDA DERIVADA \\
\hline 0,01 & $0,063 \pm 1,1 \times 10-2$ & $-2,89 \times 10-3 \pm 2,25 \times 10-4$ \\
\hline 0,12 & $0,430 \pm 2,9 \times 10-2$ & $-8,27 \times 10-3 \pm 1,77 \times 10-3$ \\
\hline 0,3 & $0,993 \pm 5,0 \times 10-2$ & $-2,11 \times 10-2 \pm 2,02 \times 10-3$ \\
\hline 0,61 & $1,600 \pm 3,2 \times 10-2$ & $-3,03 \times 10-2 \pm 1,99 \times 10-3$ \\
\hline 1,21 & $1,748 \pm 6,2 \times 10-2$ & $-2,42 \times 10-2 \pm 3,81 \times 10-3$ \\
\hline
\end{tabular}

Tabla 2 Resultados obtenidos para las mediciones de absorbancia directa y derivativa de segundo orden a una longitud de onda de $332 \mathrm{~nm}$.

A partir de estos datos, se han realizado las gráficas correspondientes a ambos tipos de mediciones (considerando que se tuvieron en cuenta los valores absolutos de aquellos correspondientes a las segundas derivadas). Los gráficos obtenidos por medio del empleo del software GraFit pueden verse en las figuras 4 y 5 respectivamente. 


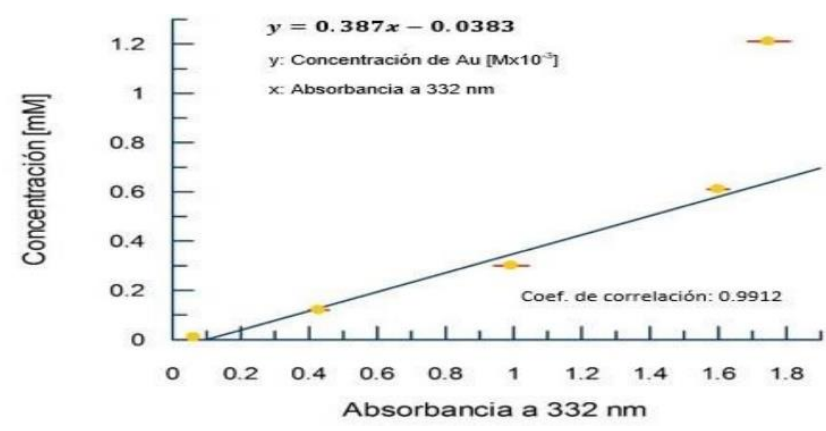

Figura 4: Concentración de Au [mM] vs. Absorbancia detectado a $332 \mathrm{~nm}$.

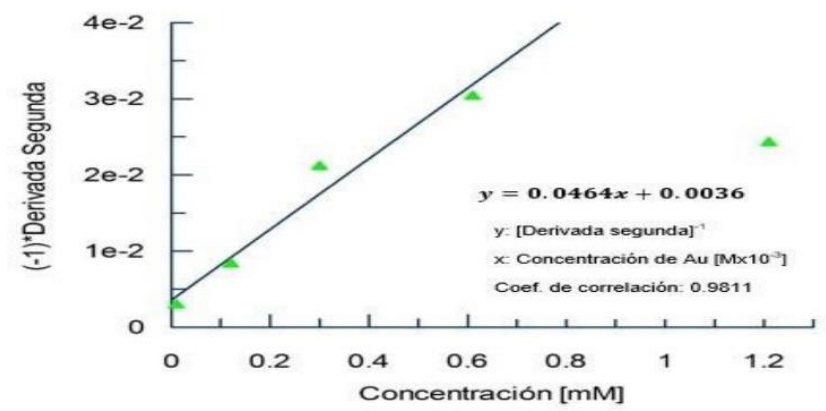

Figura 5: Derivada segunda de las medidas de absorbancia en torno a $332 \mathrm{~nm}$ Vs. Concentración de Au [mM]

\section{Experiencias de carga y descarga con carbón activado.}

Mientras se llevaba a cabo la carga del carbón activado, se fueron tomando muestras de concentración de Au a distintos tiempos. Esto mismo se ha repetido en la desorción. Los resultados se muestran en las figuras 6 y 7 respectivamente.

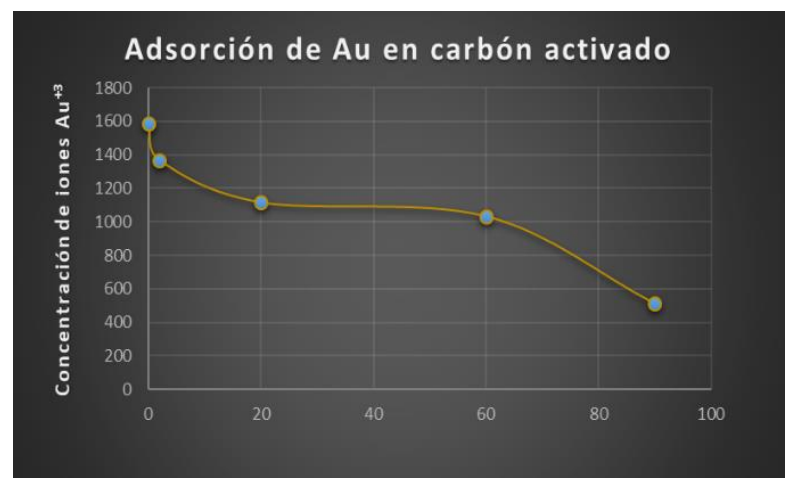

Figura 6: Concentraciones de iones $A u^{3+}$ en la carga vs. Tiempo en minutos.

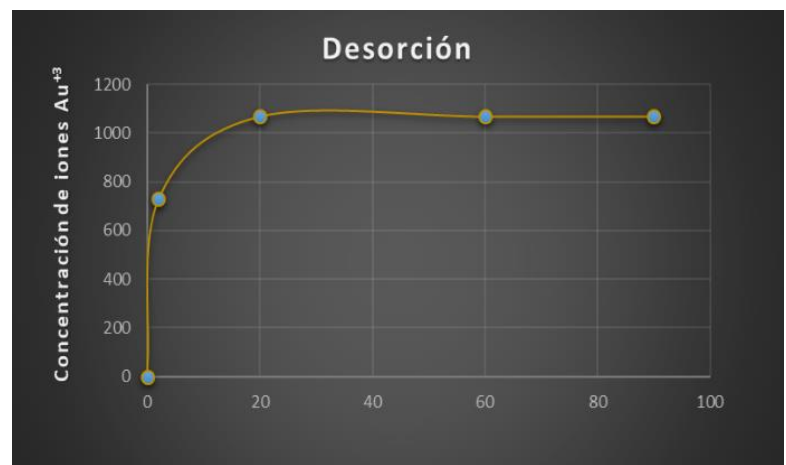

Figura 7: Concentraciones de iones $\mathrm{Au}^{3+}$ en la descarga vs. Tiempo en minutos 


\section{Conclusiones/investigaciones futuras.}

a) Mediante la espectrofotometría UV/Vis a $332 \mathrm{~nm}$ pudo calibrarse una curva para la determinación cuantitativa de Au en solución. Tal curva obedece la Ley de Beer para bajas concentraciones [0,09-0,6 mM].

b) La obtención de la curva permitió establecer un método de análisis cuantitativo para Au en muestras provenientes de chatarra electrónica.

c) El empleo de TSA en la lixiviación de Au demuestra ser más provechoso que la tiourea. No obstante, se planean experiencias comparativas futuras en la extracción desde ambos tipos de soluciones, por medios electroquímicos.

d) Se ha logrado una adsorción de Au del $68 \%$ a temperatura ambiente y pH comprendido entre 10,5 y 12. La modificación del pH a valores por debajo de 9,5, favorece la desorción de Au en soluciones de TSA. La temperatura óptima para la desorción fue de $40^{\circ} \mathrm{C}$.

e) Actualmente se está diseñando una cuba adecuada para llevar a cabo los procedimientos electrolíticos de extracción.

\section{Agradecimientos}

Al Dr. Roger López Padilla, por introducirme en esta apasionante temática.

A la directora y co-director, Dra. Mariana Minchiotti y Dr. Roberto Lucci, por su predisposición, gestión y colaboración a lo largo de todo el desarrollo.

A los todos becarios y cuerpo docente del Departamento de Ingeniería Metalúrgica que han participado y a los que actualmente me acompañan y me ayudan en este camino.

A la Facultad de Ciencias Agropecuarias de la Universidad Nacional de Córdoba, en cuyos laboratorios he trabajado.

Al Ing. Pedro Delvasto, que desde la Escuela de Ingeniería Metalúrgica y Ciencia de Materiales de la Universidad Industrial de Santander (Colombia) me ha dado gran soporte en mis investigaciones.

A la Dra. Andrea Díaz del Instituto de Ingeniería en Minas (San Juan) y a todos los colegas conocidos de la Facultad de Ingeniería de la Universidad Nacional de San Juan, por su apoyo y hospitalidad brindada en cada curso o trabajo realizado en conjunto.

Al Consejo Nacional de Investigaciones Científicas y Técnicas (CONICET), por el financiamiento otorgado a través de sus programas de becas.

\section{Referencias}

Arda Ișıldara, Eldon R. Renea, Eric D. van Hullebuscha, Piet N.L. Lensa. Electronic waste as a secondary source of critical metals: Management and recovery technologies. 2018.

Camelino, S., Rao, J., López Padilla. Initial studies about gold leaching from Printed Circuit Boards. Procedia Materials Science, 2015.

Camelino, S., Minchiotti M.1,2, Bariles R.1, López Padilla R.1, Colazo, J. Optimización de un procedimiento para la determinación de oro mediante espectrofotometría UV/Vis. Revista Materia, Rio de Janeiro, 2018.

Dreisinger, D., Molleman, E. The treatment of copper-gold ores by ammonium thiosulfate leaching. Hydrometallurgy 66, 2002.

Örgül, S., Atalay. Ü. Reaction chemistry of gold leaching in thiourea solution for a Turkish gold ore. Hydrometallurgy 67, 2002.

Roca Vallmajor, A., González Lara, J. M., Patiño Cardona, F., Cruells Cadevall, M. Oxidation of thiosulfate with oxygen using copper (II) as a catalyst. Metals 2019, 9,387.

Vargas C., Navarro P., Aguayo C. Adsorción de $\mathrm{Au}(\mathrm{CN})^{2-}$ en carbón activado. Efecto de variables y análisis mecanístico. CONAMET/SAM-2008

Vargas C., Navarro P., Alonso M., Alguacil FJ. The adsorption of Gold on Activated Carbon from ThisulfateAmmoniacal Solutions. Gold bulletin, September 2006. 